\title{
Sleep Quality, Depression, Hopelessness, and Quality of Life in Elderly Hemodialysis Patients
}

\author{
(1) Funda Datlı Yakaryılmaz', (1) İrem Pembegül2 \\ 1 Inönü University Faculty of Medicine, Department of Geriatrics, Malatya, Turkey \\ 2Turgut Özal University Faculty of Medicine, Department of Nephrology, Malatya, Turkey
}

\begin{abstract}
Objective: This study aimed to assess and compare the relationships between depression, hopelessness, sleep, and quality of life in two age groups of elderly and young patients with end-stage renal disease undergoing hemodialysis (HD).

Materials and Methods: The study included 130 patients under follow-up for hemodialysis ( 55 aged $<60$ years and 75 aged $\geq 60$ years). Depression levels, sleep quality, and quality of life of all participants were evaluated using the Beck depression inventory (BDI), Beck hopelessness scale (BHS), the Pittsburgh sleep quality index, and the short form-36, respectively.

Results: Of the participants, $55 \%(n=72)$ were female, and $45 \%(n=58)$ were male. The mean age was $59.48 \pm 14.57$ years. There was no significant difference between the age groups concerning the BHS scores $(6.82 \pm 4.73$ vs. $6.57 \pm 4.20)(p=0.756)$. However, the $B D I$ scores were significantly higher among younger participants $(38.67 \pm 19.45$ vs. $23.45 \pm 17.13)(p<0.001)$. Also, the sleep quality of the elderly group was significantly worse than the younger group $(5.16 \pm 2.93 \pm 6.49 \pm 3.01)(p=0.013)$. Concerning the health-related quality of life, physical performance $(45.81 \pm 19.33$ vs. $39.49 \pm 13.07)$ and mental health $(44.63 \pm 18.69$ vs. $36.80 \pm 16.49)$ subscales were significantly lower in the elderly group $(p=0.028$ and $p=0.013$, respectively.
\end{abstract}

Conclusion: We conclude that age is a significant factor requiring consideration when assessing and managing patients under HD. Although the functional capacity and sleep quality deteriorate with age, younger HD patients are more disadvantaged concerning the possibility of depression. Thus, we suggest age-specific approaches in HD patients with a multidisciplinary team.

Keywords: Sleep, quality of life, depression, hope, elderly, hemodialysis

\section{Introduction}

Chronic renal failure is a well-known public health problem, which can result in end-stage renal disease (ESRD) and necessitates renal replacement therapies such as renal transplantation or hemodialysis (HD)/peritoneal dialysis (1). The availability of HD significantly increased in the last decade. However, the prevalence of ESRD also increased and reached 11$13 \%$ (2). This rise is related not only to changes in demography and aging but also to the increase in co-morbid diseases such as diabetes and hypertension (3).

Like other chronic diseases, ESRD deteriorates life quality and increases the incidence of psychopathological conditions compared to the normal population (4). The prevalence of depression is about $2-10 \%$ in the general population. However, it may reach $23-29 \%$ in people with chronic renal failure $(5,6)$. Despite the escalation of depression in this population, there is no report investigating its relationship to hope and suicidal tendencies in patients on maintenance dialysis.

On the other hand, sleep is a dynamic condition with significant influences on daily functions, including mental and physical health (7). Poor sleep quality may even disturb emotions and thoughts. Patients with poor sleep quality have a poor quality of life with many physical or emotional symptoms, such as concentration difficulties, tiredness, decreased pain tolerance, loss of appetite, depression, and anxiety (8). The survival rates in ESRD patients have increased in relation to renal replacement

Address for Correspondence: Funda Datı Yakaryılmaz, İönü University Faculty of Medicine, Department of Geriatrics, Malatya, Turkey E-mail: fundadatli@yahoo.com ORCID: orcid.org/0000-0001-5633-0939

Received: 25.01.2022 Accepted: 16.02.2022

Cite this article as: Datı Yakaryılmaz F, Pembegül I. Sleep Quality, Depression, Hopelessness, and Quality of Life in Elderly Hemodialysis Patients. Eur J Geriatr Gerontol 2022;4(2):97-102

๑Copyright 2022 by the Academic Geriatrics Society / European Journal of Geriatrics and Gerontology published by Galenos Publishing House. 
therapies and other therapeutic interventions, which increased the significance of evaluating health-related quality of life (HROoL) in these patients (9).

Given the extended life expectancy in ESRD patients, the follow-up of quality of life, depression, hopelessness, and sleep becomes more important in elderly patients.

This study aimed to assess and compare the relationships between depression, hopelessness, sleep, and quality of life in two age groups of elderly and young patients with ESRD.

\section{Materials and Methods}

\section{Study design}

The study was conducted in a cross-sectional design. All participants gave written individual informed consent to participate. The study protocol was approved by the Local Ethics Committee at Malatya Turgut Özal University Medical Faculty and conducted according to the criteria of the Helsinki Declaration. Informed consent was obtained from all the patients included in the study.

\section{Setting}

This study was conducted at the department of nephrology at Malatya Training and Research Hospital from July 2018 to January 2019. Established in 1939, the study hospital currently provides secondary-level healthcare services in eastern Turkey with 1.040 inpatient beds, 240 outpatient clinics, 23 surgical intervention rooms, a 90-bed capacity intensive care unit, and a HD unit able to serve 29 simultaneous patients.

\section{Participants}

Patients $>18$ years of age with ESRD, who were receiving HD for at least 3 months were included in the study. The exclusion criteria of the study were patients not able to complete the study questionnaire due to cognitive impairment $(n=2)$, active psychosis $(n=1)$, a history of recent hospitalization $(n=2)$, history of malignancy $(n=1)$. Data could be collected from 130 patients out of the 150 patients under treatment (Figure 1). All patients under follow-up were invited to join the study without sampling.

\section{Variables}

Age, gender, marital status, cigarette smoking, dialysis access, dialysis duration, body mass index (BMI), hemoglobin, albumin, C-reactive protein, ferritin, intact parathyroid hormone (iPTH), lipid parameters, and serum 25-hydroxyvitamin D [25(OH) D] were assessed in all patients. Blood samples for laboratory parameters were collected on the day of the survey. Blood samples were taken from the patients just before the dialysis session. Serum $25(\mathrm{OH})$ D levels were measured by liquid chromatography-tandem mass spectroscopy (Agilent Technologies, Santa Clara, CA, USA), the measurement of iPTH

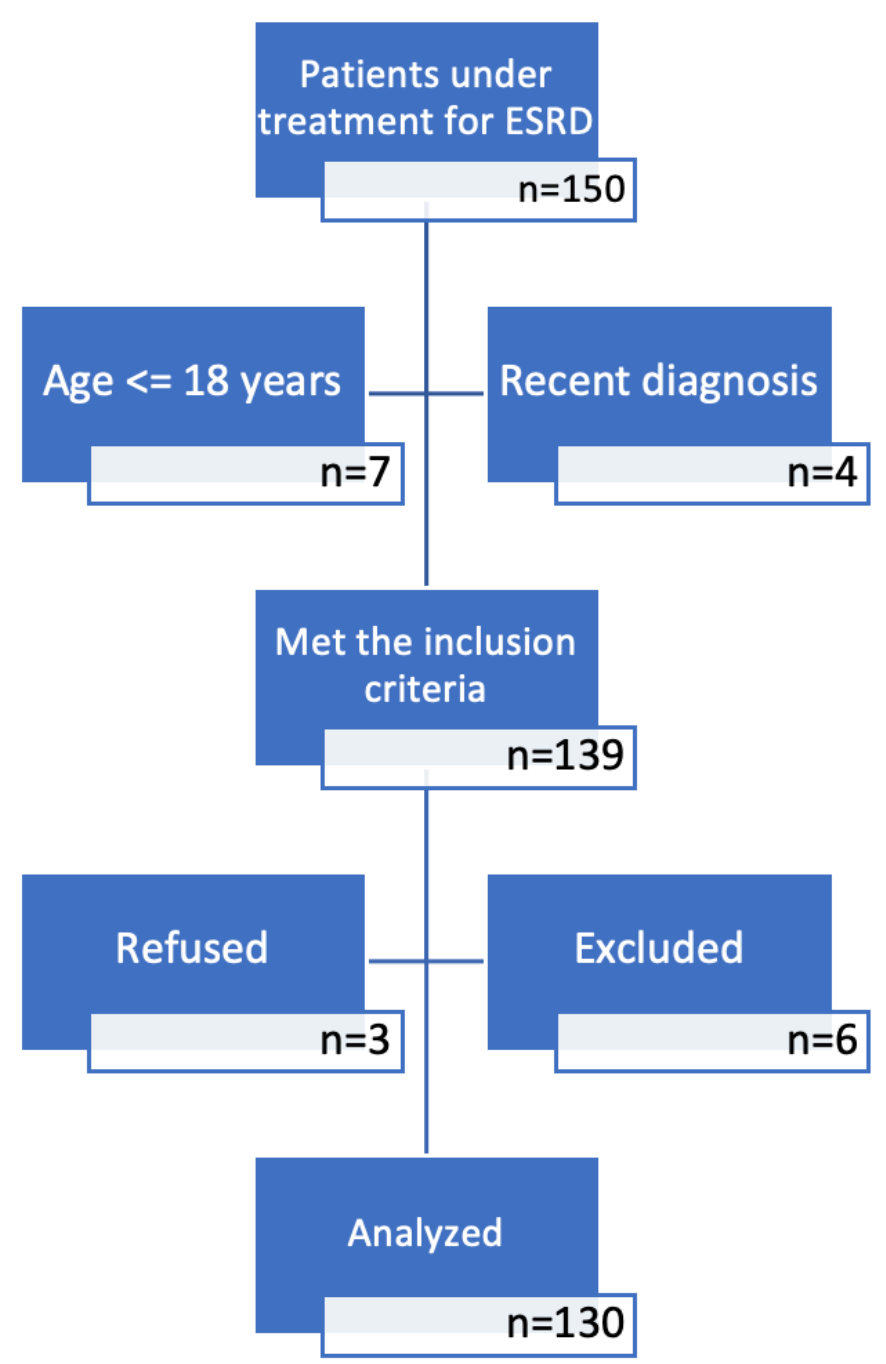

Figure 1. Study flow diagram

levels was made by the chemiluminescence method (ADVIA centaur XPT immunoassay, Siemens, Erlangen, Germany) $(10,11)$. Ferritin levels were measured by ferritin immunoassay (ADVIA Centaur XPT immunoassay, Siemens, Erlangen, Germany), hemoglobin was measured by fluorescence flow cytometry (Sysmex XN 2000, Norderstedt, Germany) $(12,13)$. Albumin and lipid parameters were measured by spectrophotometry (Beckman Coulter AU 2700, Krefeld, Germany) (14). C-reactive protein was measured by the immunonephelometric method (NFL BN-II, Erlangen, Germany) (15).

\section{Short form-36 health survey (SF-36)}

The SF-36 consists of eight dimensions, generating a profile of HROoL (16). These dimensions are: 1) Physical functioning, 2) Role limitations due to physical functioning, 3) Bodily pain, 4) General health perceptions, 5) Vitality, 6) Social functioning, 7) Role limitations due to emotional functioning, and 8) Mental health. Raw scores are transformed into a score between zero and a hundred for each dimension. Higher scores indicate better health. 


\section{Beck's depression inventory (BDI) and the Beck hopelessness scale (BHS)}

To facilitate the investigation of despair in various psychopathological situations, Beck has established a tool designed to reflect the negative expectations of the participants (17). The BHS and the BDI were used in this study. The BHS has 20 true/false statements, of which 9 are false, and 11 are correct. Each correct answer is scored as 1, calculating the total "hopelessness score," giving a score range between 0 and 20 . The cut-off points are categorized as follows: 0-4: Minimum hopeless, 5-8: Mild hopeless, 9-13: Moderately hopeless, and 14-20: Severe hopeless. Additionally, we defined a score of $\geq 9$ as a suicide predictor in people with serious diseases (4). On the other hand, BDI has questions that describe attitudes and symptoms concerning depression. It comprises 21 groups of statements, and each group investigates a type of depression symptom. The higher the score displayed, the greater is the intensity of symptoms. Cut-off points used for BDI are defined as follows: Minimal depression: 0-11, mild depression: 12-19, light depression: 20-35, and severe depression: 36 and above (18).

\section{Sleep quality}

The Pittsburgh sleep quality index (PSOI) was used to assess sleep quality. The standard (past month) version was used at the screening visit, and a modified (past week) version was used at all subsequent administrations. The scores ranged from 0 to 21, with higher values indicating poorer sleep quality (19). A score higher than 5 indicates poor sleep quality.

\section{Functional capacity}

Patients' functional dependency was assessed by the activities of daily living (ADL) and Instrumental Activities of Daily Living (IADL) tests. The Barthel index of ADL was used for evaluating physical disabilities (20). This scale includes dressing, bathing, grooming, using the toilet, eating, transferring, and incontinence. Scores can range from 0 to 100, and higher scores indicate independence. On the other hand, the Lawton index was used to evaluate the disability in IADL. This scale aims to find out subject performance in the following activities: Doing laundry, shopping, taking medicines, housekeeping, food preparation, using the telephone, using transportation, and managing money (21). Higher scores indicate higher independence according to this scale.

\section{Bias}

All patients under follow-up were invited to join the study to prevent selection bias. Furthermore, the data collection was done by the same researcher to decrease measurement bias.

\section{Statistics}

Data were analyzed using the Statistical Package for the Social Sciences (SPSS) version 25.0 software (SPSS Inc., Chicago, IL, USA). Independent samples t-test and chi-square tests were used to compare numerical and categorical variables between the age groups. Besides, the Pearson's correlation test was used to identify if there was any correlation between the changes in outcomes (i.e., if the change scores in depression, sleep, and quality of life outcomes correlated with each other). The threshold for statistical significance was set to $p<0.05$.

\section{Results}

Data of 130 participants were analyzed. While $55 \%(n=72)$ of patients were female, $45 \%(n=58)$ were male. The mean age was $59.48 \pm 14.57$ years. Patients were divided to two groups based on age: $\geq 60$ and $<60$ years. There was no significant difference between the two groups regarding HD duration and BMI. However, when the laboratory results were evaluated, PTH was high in the young age group, while vitamin D levels were found to be significantly low ( $p=0.006, p=0.047$ : Respectively) (Table 1). Also, functional capacity assessment, ADL and AIDL were significantly higher in the younger group (Table 2).

The total BHS score ranged from 1 to 19 in the whole group, with a median of 5 points. According to the BHS, 56 (43.1\%) patients had minimal, 40 (30.8\%) light, 19 (14.6\%) moderate, and $15(11.5 \%)$ had severe hopelessness symptoms. When young and old HD patients were compared, there was no significant difference between the scores (Table 2).

BDI scores were significantly higher among the younger participants (Table 2). The BDI score ranged from 4 to 60, with a median score of 29.8. According to the cut-off scores, the proportions of mild, moderate, and severe depression were $18.5 \%(n=24), 23.8 \%(n=31)$, and $35.4 \%(n=46)$, respectively. Of the patients, $78(60.0 \%)$ had poor sleep quality. The sleep quality of the elderly group was significantly worse than the younger group (Table 2).

BHS showed a positive correlation with the BDI $(r=0.364$, $p<0.001)$ and a negative correlation with the Barthel index of ADL $(r=-0.173 p=0.049)$. On the other hand, PSOl positively correlated with age $(r=0.282, p=0.001)$ and BMI $(r=0.249$, $\mathrm{p}=0.004)$.

Concerning the HROoL, physical performance, and mental health subscales were significantly lower in the elderly group (Table 3).

\section{Discussion}

In our study, ESRD HD patients aged 60 years and older had lower functional capacity and depression, similar hopelessness, and poorer sleep quality than similar patients aged less than 60 


\begin{tabular}{|c|c|c|c|}
\hline & Young $(n=55)$ & Older $(n=75)$ & p \\
\hline \multicolumn{4}{|l|}{ Sex n (\%) } \\
\hline Women & $34(61.8 \%)$ & $38(50.7 \%)$ & 0.206 \\
\hline Men & $21(32.2 \%)$ & $37(49.3 \%)$ & \\
\hline Under dialysis since (months) median (min-max) & $48(2-204)$ & $44(4-204)$ & 0.786 \\
\hline BMI $\left(\mathrm{kg} / \mathrm{m}^{2}\right)$ & $24.82(17.26-36.0)$ & $24.50(16.00-42.8)$ & 0.913 \\
\hline \multicolumn{4}{|l|}{ Laboratory values } \\
\hline Urea (mg/dL) & $135(83-216)$ & $130(67-219)$ & 0.882 \\
\hline Creatinine (mg/dL) & $8.25(2.8-18.25)$ & $7.20(2.25-13.88)$ & 0.072 \\
\hline Uric acid $(\mathrm{mg} / \mathrm{dL})$ & $6.4(4.3-10.9)$ & $6.3(4.8-8.90)$ & 0.316 \\
\hline Total protein $(\mathrm{g} / \mathrm{dL})$ & $7(5.2-8.2)$ & 7. $(5.5-8.2)$ & 0.530 \\
\hline Albumin (g/dL) & $3.6(2.0-4.4)$ & $3.6(2.0-4.4)$ & 0.758 \\
\hline Calcium (mg/dL) & $8.8(7.2-9.9)$ & $8.8(7.8-11.5)$ & 0.442 \\
\hline Phosphorus (mg/dL) & $5(2.1-9.3)$ & $4.5(3-9.1)$ & 0.218 \\
\hline Parathormone (PTH) (pg/mL) & $447.9(14.52-1881)$ & $354(54.95-1228)$ & $0.022^{*}$ \\
\hline Vitamin $D(n g / m L)$ & $8.47(3-54.46)$ & $11.71(3-93.43)$ & $0.049^{*}$ \\
\hline C-reactive protein (mg/L) & $0.54(0.003-3.81)$ & $0.79(0.04-14.67)$ & 0.272 \\
\hline Ferritin $(\mathrm{mL} / \mathrm{ng})$ & $600.87 \pm 425.62$ & $570.12 \pm 350.58$ & 0.917 \\
\hline Hemoglobin (g/dL) & $11.2(7.4-14.4)$ & $11.10(7.20-14.6)$ & 0.567 \\
\hline
\end{tabular}

\section{Table 2. Functional capacity, depression, and sleep quality assessment of the groups}

\begin{tabular}{|l|l|l|l|}
\hline $\begin{array}{l}\text { Functional } \\
\text { assessment }\end{array}$ & Young $(\mathbf{n = 5 5 )}$ & Older $(\mathbf{n = 7 5 )}$ & $\mathbf{p}$ \\
\hline Barthel ADL & $100(15-100)$ & $75(10-100)$ & $<0.001^{*}$ \\
\hline Lawton brody IADL & $5(1-8)$ & $3(1-8)$ & $<0.001^{*}$ \\
\hline BDI & $6(1-18)$ & $5(1-19)$ & $<0.001^{*}$ \\
\hline BHS & $36(8-63)$ & $17(4-63)$ & 0.915 \\
\hline PSOI & $4(1-14)$ & $5(1-13)$ & $0.003^{*}$ \\
\hline
\end{tabular}

Data are presented as mean \pm standard deviation. $A D L$ : Activities of daily living, IADL: Instrumental activities of daily living, BDI: Beck depression inventory, BHS: Beck hopelessness scale, PSOI: Pittsburgh sleep quality index, ${ }^{*} p<0.05$ significant

Table 3. Health-related quality of life assessment of the age groups

\begin{tabular}{|l|l|l|l|}
\hline SF-36 & Young $(\mathbf{n = 5 5})$ & Older $(\mathbf{n = 7 5 )}$ & $\mathbf{p}$ \\
\hline PF & $45.81 \pm 19.33$ & $39.49 \pm 13.07$ & $0.028^{*}$ \\
\hline SF & $44.82 \pm 16.98$ & $41.94 \pm 15.29$ & 0.313 \\
\hline PR & $17.54 \pm 22.35$ & $11.17 \pm 16.11$ & 0.061 \\
\hline ER & $12.021 \pm 16.23$ & $12.62 \pm 14.14$ & 0.824 \\
\hline MH & $44.63 \pm 18.69$ & $36.80 \pm 16.49$ & $0.013^{*}$ \\
\hline V & $29.21 \pm 18.52$ & $24.54 \pm 14.44$ & 0.109 \\
\hline P & $37.40 \pm 17.35$ & $34.48 \pm 14.31$ & 0.295 \\
\hline GH & $28.18 \pm 16.66$ & $23.66 \pm 13.49$ & 0.091 \\
\hline Da & & &
\end{tabular}

Data are presented as mean \pm standard deviation. PF: Physical functioning, SF: Social functioning, PR: Physical role limitations, ER: Emotional role limitations, MH: Mental health, V: Vitality, P: Pain, GH: General health, ${ }^{*} p<0.05$ significant years. However, there was no significant difference between the two age groups regarding the quality of life, except for physical functioning and mental health. Also, there was no significant difference in the BHS scores concerning the age groups. Furthermore, both the BHS and PSOI showed some correlations with the other variables.

The prevalence of geriatric depression increases rapidly with the steady aging of the population and the increase in chronic diseases with advancing age (22). Depression has been reported in up to $70 \%$ of HD patients $(23,24)$. Tsevi et al. $(25)$ reported that the occurrence of depression was associated with the duration of HD in patients undergoing HD treatment. In another study, it was emphasized that depression was associated with various markers of HD proficiency, such as high blood pressure, serum albumin concentration, and serum creatinine concentration. However, in these studies, patients were not grouped by age (24).

Despite the well-known strong relationship between hopelessness and depression, our study reveals that hopelessness and depression are partly different phenomena. The hopelessness levels in the young and old groups were close to each other, while the depression rate was significantly higher in the young group (26). Similarly, Andrade et al. (4) found a high prevelance of hopelessness, suicidal ideation, and depression symptoms in HD patients. In our study, in the despair assessment according to age, BHS values were very close to each other, but it is surprising that $\mathrm{BDI}$ values were higher in younger HD patients. A possible 
explanation for this finding could be that younger people affected by a debilitating chronic disease are more vulnerable because of their impossibility to accomplish plans for their life. Despite the fact that depression is a common disorder in the general elderly population, younger HD patients should be regularly evaluated for depression.

Most HD patients suffer from poor sleep quality. Our study showed that $60 \%$ of HD patients had poor sleep quality. Furthermore, there was also a correlation between sleep quality and age as well as BMI. Likewise, other studies have verified a significant relationship between sleep quality and increasing age $(27,28)$.

Increased age-related changes (physical limitations, use of various medications, and lifestyle changes) appear to affect the sleep quality of HD patients. It has been shown in several studies that patients receiving HD treatment have significantly poorer sleep quality compared to the general population $(29,30)$. We found that the majority of patients receiving HD treatment had poor sleep quality. Additionally, we identified an association between poor sleep quality with age and BMI. Hydarinia Naieni et al. (31) reported that sleep quality was significantly associated with age in HD patients, while another study showed that sleep quality had a significant relationship with BMI and diabetes (32). According to our study, quality of life was significantly lower in the elderly group with sleep disorders. Pain, depression, and sleep disorders due to chronic diseases cause a decrease in quality of life. In our study, physical performance and mental health subscale scores of the SF-36 were significantly lower in the elderly group. Similar to our study, Zouari et al. (33) emphasized that being 60 years or older is associated with a poor quality of life.

The aging population and the increase in the number of patients suffering from chronic diseases highlight the imperative links between mental, social and physical health $(4,26,29)$. In addition, thanks to advances in the treatment of chronic kidney disease, many patients can live to old age. In recent years, it has been noticed that many nephrologists actually act as "amateur geriatric specialists", especially by the American and United Kingdom nephrology associations, and steps have been taken to improve kidney education in this area (34). Both specialties are very familiar with the concept of patient-centered care. However, renal services usually focus on holistic symptom control, dietary changes, and the psychological impact of kidney disease, while geriatric comprehensive assessment focuses on functional status, cognitive impairment, and multiple drug use and aims to maintain or improve the quality of life and prevent it future readmissions (35-37). However, there is no specialization in this field in our country. Therefore, the treatment of HD patients should be organized by a multidisciplinary team consisting of nephrologists, geriatricians and psychiatrists.

\section{Study Limitations}

Our study should be interpreted in light of some limitations. It is a descriptive study in a local sample with limited generalizability of the findings. Multi-center studies with control groups are needed to verify the results.

\section{Conclusion}

We conclude that age is a significant factor requiring consideration when assessing and managing patients under HD. Although the functional capacity and sleep quality deteriorate with age, younger HD patients are more disadvantaged concerning the possibility of depression. Thus, we suggest agespecific approaches in HD patients with a multidisciplinary team.

\section{Ethics}

Ethics Committee Approval: The study protocol was approved by the Local Ethics Committee at Malatya Turgut Özal University Medical Faculty (IRB number: E-23536505-604.02) and conducted according to the criteria of the Helsinki Declaration.

Informed Consent: Informed consent was obtained from all the patients included in the study.

Peer-review: Externally peer-reviewed.

\section{Authorship Contributions}

Surgical and Medical Practices: F.D.Y., I.P., Concept: F.D.Y., Design: F.D.Y., I.P., Data Collection or Processing: I.P., Analysis or Interpretation: F.D.Y., Literature Search: F.D.Y., I.P., Writing: F.D.Y., I.P.

Conflict of Interest: No conflict of interest was declared by the authors.

Financial Disclosure: The authors declared that this study received no financial support.

\section{References}

1. System USRD. 2013 USRDS 2013 annual data report: atlas of chronic kidney disease and end-stage renal disease in the United States. Natl Institutes Heal Natl Inst Diabetes Dig Dig Kidney Dis 2014.

2. Hill NR, Fatoba ST, Oke JL, Hirst JA, O'Callaghan CA, Lasserson DS, Hobbs FD. Global prevalence of chronic kidney disease-a systematic review and metaanalysis. PLoS One 2016;11:e0158765.

3. Thomas B, Wulf S, Bikbov B, Perico N, Cortinovis M, Courville de Vaccaro $K$, Flaxman A, Peterson $H$, Delossantos A, Haring D, Mehrotra R, Himmelfarb J, Remuzzi G, Murray C, Naghavi M. Maintenance dialysis throughout the world in years 1990 and 2010. J Am Soc Nephrol 2015;26:2621-2633.

4. Andrade SV, Sesso R, Diniz DH. Hopelessness, suicide ideation, and depression in chronic kidney disease patients on hemodialysis or transplant recipients. J Bras Nefrol 2015;37:55-63. 
5. Kessler RC, Berglund P, Demler 0 , Jin R, Koretz D, Merikangas KR, Rush AJ, Walters EE, Wang PS; National Comorbidity Survey Replication. The epidemiology of major depressive disorder: results from the National Comorbidity Survey Replication (NCS-R). JAMA 2003;289:3095-3105.

6. Palmer $\mathrm{S}$, Vecchio $\mathrm{M}$, Craig JC, Tonelli M, Johnson DW, Nicolucci A, Pellegrini F, Saglimbene V, Logroscino G, Fishbane S, Strippoli GF. Prevalence of depression in chronic kidney disease: systematic review and meta-analysis of observational studies. Kidney Int 2013;84:179-191.

7. João KADR, Becker NB, de Neves Jesus $\mathrm{S}$, Martins RIS. Validation of the Portuguese version of the Pittsburgh sleep quality index (PSOI-PT). Psychiatry Res 2017;247:225-259.

8. Tel $H_{1}$ Tel $H_{1}$ Esmek M. Quality of sleep in hemodialysis patients. Dial Transplant 2007;36:479-484.

9. Liem YS, Bosch $J \mathrm{~L}$, Arends LR, Heijenbrok-Kal MH, Hunink MGM. Quality of life assessed with the Medical Outcomes Study Short Form 36-Item Health Survey of patients on renal replacement therapy: a systematic review and meta-analysis. Value Health 2007;10:390-397.

10. Farrell CJ, Martin S, McWhinney B, Straub I, Williams P, Herrmann M. Stateof-the-art vitamin D assays: a comparison of automated immunoassays with liquid chromatography-tandem mass spectrometry methods. Clin Chem 2012;58:531-542.

11. Kuczera P, Maszczyk A, Machura E, Kurzak E, Adamczak M, Więcek A. Serum parathyroid hormone concentrations measured by chemiluminescence and electrochemiluminescence methods - are the results comparable in haemodialysis patients with chronic kidney disease? Endokrynol Pol 2015:66:219-223.

12. Karakochuk CD, Whitfield KC, Rappaport Al, Barr SI, Vercauteren SM, McLean J, Hou K, Talukder A, Houghton LA, Bailey KB, Boy E, Green TJ. Comparison of four immunoassays to measure serum ferritin concentrations and iron deficiency prevalence among non-pregnant Cambodian women and Congolese children. Clin Chem Lab Med 2017;55:65-72.

13. Wojda U, Noel P, Miller JL. Fetal and adult hemoglobin production during adult erythropoiesis: coordinate expression correlates with cell proliferation. Blood 2002;99:3005-30013.

14. Sözgen $K$, Cekic SD, Tütem E, Apak R. Spectrophotometric total protein assay with copper(II)-neocuproine reagent in alkaline medium. Talanta 2006;68:1601-1609.

15. Verbeke $F$, Lambrecht $S$, Lecocq $E$, Parez JJ, Delanghe J. Increased C-reactive protein values in the absence of inflammation: monoclonal immunoglobulin interference in immunonephelometry. Clin Chem Lab Med 2019;57:e311-e313.

16. Ware Jr JE, Sherbourne CD. The MOS 36-item short-form health survey (SF36): I. Conceptual framework and item selection. Med Care 1992;30:473483.

17. Beck AT, Weissman A, Lester D, Trexler L. The measurement of pessimism: the hopelessness scale. J Consult Clin Psychol 1974;42:861-865.

18. Beck AT, Weishaar ME. Suicide risk assessment and prediction. Crisis 1990;11:22-30

19. Buysse DJ, Reynolds III CF, Monk TH, Berman SR, Kupfer DJ. The Pittsburgh Sleep Quality Index: a new instrument for psychiatric practice and research. Psychiatry Res 1998;28:193-213.

20. Mahoney $\mathrm{Fl}$, Barthel DW. Functional evaluation: the barthel index. MD State Med J 1965;14:61-65.
21. Lawton MP, Brody EM. Assessment of older people: self-maintaining and instrumental activities of daily living. Gerontologist 1969;9:179-186.

22. Moussavi S, Chatterji S, Verdes E, Tandon A, Patel V, Ustun B. Depression, chronic diseases, and decrements in health: results from the World Health Surveys. Lancet 2007;370:851-858.

23. Coulibaly G, Goumbri P, Ouédraogo N, Dabilgou A, Napon C, Karfo K, Ouango $J G$, Lengani $A$, Ouédraogo $A$. [Factors associated with depressive symptoms in chronic hemodialysis patients of centre hospitalier universitaire Yalgado Ouedraogo (Burkina Faso)]. Nephrol Ther 2016;12:210-214.

24. Sqalli-Houssaini T, Ramouz I, Fahi Z, Tahiri A, Sekkat FZ, Ouzeddoun N, Ezzaitouni F, Benamar L, Rhou H, Ktiouet JE, Balafrej L. [Effects of anxiety and depression on haemodialysis adequacy]. Nephrol Ther 2005;1:31-37.

25. Tsevi MY, Salifou S, Sabi AK, Noto-Kadou-Kaza B, Amekoudi EY, Dassa SK. [Depression in patients on chronic hemodialysis at the Sylvanus Olympio University Hospital of Lomé (Togo)]. Pan Afr Med J 2016;25:26.

26. Haatainen KM, Tanskanen A, Kylmä J, Antikainen R, Hintikka J, Honkalampi $\mathrm{K}$, Koivumaa-Honkanen $\mathrm{H}$, Viinamäki $\mathrm{H}$. Life events are important in the course of hopelessness-a 2-year follow-up study in a general population. Soc Psychiatry Psychiatr Epidemiol 2003;38:436-441.

27. Menon VB, Alla P, Madhuri S, Sanathan SR, Shetty MS, Ram D. Sleep quality in end-stage renal disease patients on maintenance hemodialysis: A six month prospective survey. Int J Pharm Sci Res 2015;6:660-668.

28. Trbojević-Stanković J, Stojimirović B, Bukumirić $Z_{1}$ Hadzibulić $E_{1}$ Andrić $B$ Djordjević V, Marjanović Z, Birdjozlić F, Nesić D, Jovanović D. Depression and quality of sleep in maintenance hemodialysis patients. Srp Arh Celok Lek 2014;142:437-443.

29. Zubair U Bin, Butt B. Assessment of quality of sleep and its relationship with psychiatric morbidity and socio-demographic factors in the patients of chronic renal disease undergoing hemodialysis. J Coll Physicians Surg Pak 2017;27:427-431

30. Čengić B, Resić $H$, Spasovski G, Avdić E, Alajbegović A. Quality of sleep in patients undergoing hemodialysis. Int Urol Nephrol 2012;44:557-567.

31. Hydarinia Naieni Z, Nobahar M, Ghorbani R. The relationship between sleep quality in patients' undergoing hemodialysis at different therapeutic'shifts. Koomesh 2016;18:42-50.

32. Mehrabi S, Sarikhani S, Roozbeh J. Sleep quality in patients undergoing long-term hemodialysis using the Pittsburgh sleep quality index. Nephrourol Mon 2017:9:e44278.

33. Zouari L, Omri S, Turki S, Maâlej M, Charfi N, Ben Thabet J, Mahfoudh H, Hachicha J, Maâlej M. Quality of life in chronic hemodialysis patients: about 71 cases. Tunis Med 2016;94:40-45.

34. Oreopoulos DG, Dimkovic N. Geriatric nephrology is coming of age. J Am Soc Nephrol 2003;14:1099-1101.

35. Wiggins J, Bitzer M. Geriatric assessment for the nephrologist. Semin Dial 2012;25:623-627.

36. Conroy SP, Ansari K, Williams M, Laithwaite E, Teasdale B, Dawson J, Mason S, Banerjee J. A controlled evaluation of comprehensive geriatric assessment in the emergency department: the 'Emergency Frailty Unit'. Age Ageing 2014;43:109-114.

37. Conroy SP, Stevens T, Parker SG, Gladman JR. A systematic review of comprehensive geriatric assessment to improve outcomes for frail older people being rapidly discharged from acute hospital: 'interface geriatrics'. Age Ageing 2011;40:436-443. 